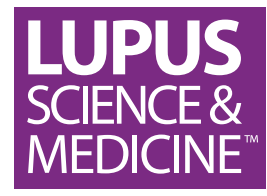

\title{
Increased von Willebrand factor levels in patients with systemic lupus erythematosus reflect inflammation rather than increased propensity for platelet activation
}

\author{
Johannes C Nossent, ${ }^{1}$ Warren D Raymond, ${ }^{2}$ Gro Østli Eilertsen ${ }^{3}$
}

To cite: Nossent JC, Raymond WD, Eilertsen GØ. Increased von Willebrand factor levels in patients with systemic lupus erythematosus reflect inflammation rather than increased propensity for platelet activation. Lupus Science \& Medicine 2016;3: e000162. doi:10.1136/lupus2016-000162

Received 14 April 2016 Revised 19 July 2016 Accepted 21 July 2016

\section{(a) CrossMark}

${ }^{1}$ Rheumatology Department, School of Medicine, The University of Western Australia/Consultant, Sir Charles Gairdner Hospital, Perth, Western Australia, Australia

${ }^{2}$ Rheumatology Department, School of Medicine \& Pharmacology, The University of Western Australia, Crawley, Western Australia, Australia ${ }^{3}$ Department of Clinical Medicine, UiT The Arctic University of Norway/ Consultant RNNK, Tromsø, Norway

\section{Correspondence to} Professor Johannes C Nossent; Johannes.nossent@ uwa.edu.au

\section{ABSTRACT}

Background: von Willebrand factor (VWF) is involved in platelet plug formation and protein transport. Increased VWF levels in systemic lupus erythematous (SLE) are considered risk factors for vascular events. VWF protein levels, however, do not accurately reflect its platelet-aggregating function, which has not been examined in SLE.

Methods: Cross-sectional study with clinical and laboratory data obtained in patients with SLE $(n=92)$ from a regional lupus registry. VWF function was determined by ristocetin-induced platelet aggregation (VWF ristocetin cofactor, VWF:RCo) and VWF levels by turbidimetric assay (VWF antigen, VWF:Ag). The platelet-aggregating activity per VWF unit was estimated by the VWF RCo/Ag ratio. Healthy controls served as comparators and associations were evaluated by non-parametric methods.

Results: VWF:Ag (142\% vs 107\%, $p=0.001)$ and VWF: $\mathrm{RCo}$ levels ( $123 \%$ vs $78 \%, \mathrm{p}<0.041$ ) were increased in patients with SLE, but VWF RCo/Ag ratio was similar as in controls ( 0.83 vs $0.82, p=0.8)$. VWF:Ag levels were higher in patients experiencing serositis but unrelated to other manifestations, thrombotic disease, Systemic Lupus Erythematous Disease Activity Index 2000 or Systemic Lupus International Collaborative ClinicsDamage Index. VWF:Ag levels correlated significantly with VWF:RCo levels (Rs $0.8, p<0.001$ ), erythrocyte sedimentation rate (ESR) (Rs $0.32, p<0.01)$, anti-dsDNA $\mathrm{Ab}$ (Rs 0.27, $p<0.01$ ), total IgG (Rs $0.33 p<0.01$ ), fibrinogen (Rs $0.28, p<0.01$ ) and ceruloplasmin (Rs $0.367, p<0.01$ ) levels. VWF:RCo levels were not related to clinical findings but were correlated with ESR, antidsDNA and transferrin levels. No serological associations existed for VWF RCo/Ag ratio (all $p>0.2$ ). Conclusions: In this SLE cohort, VWF:Ag behaved similarly to acute-phase reactants, but VWF:Ag increases were not matched by increases in functional activity per unit of VWF. Thus, more VWF did not increase the propensity for platelet aggregation in SLE.

\section{INTRODUCTION}

von Willebrand factor (VWF) is stored as single prepro-protein mainly in endothelial cells (as Weibel-Palade bodies) and to a lesser extent in megakaryocytes (in $\alpha$-granules). The secreted VWF protein is made up of a number of covalently linked subunits and the number of subunits (2-40) is an important determinant of the platelet aggregation by VWF. ${ }^{1}{ }^{2}$ Reductions in the level or functional activity of VWF protein lead to a bleeding diathesis, that is, von Willebrand's disease (VWD), while increased VWF levels have been reported in patients with acute coronary syndromes, pulmonary arterial hypertension and scleroderma. ${ }^{3-7}$ Extreme elevations in VWF levels occur in patients with thrombotic thrombocytopenic purpura, where platelet aggregation is induced by ultralarge VWF multimers that result from a failure of serine protease ADAMTS13 to cleave VWF into smaller multimers. ${ }^{8}$

Patients with systemic lupus erythematosus (SLE) are predisposed to atherothrombotic events. ${ }^{9-11}$ This increased risk is attributable to a complex interplay between endothelial strain and (non-) traditional cardiovascular risk factors. ${ }^{12-14}$ Earlier studies have reported increased VWF protein levels in patients with SLE $^{15-17}$ and in some studies VWF was a risk factor for future cardiovascular events. ${ }^{18}$ However, it remains unclear whether VWF is causally related to the occurrence of arterial thrombosis or primarily mirrors endothelial dysfunction, promoting atherosclerosis and subsequent arterial thrombosis. ${ }^{19}$ Also, VWF levels assess the mass of circulating VWF protein, but do not accurately reflect VWF platelet-aggregating function. While VWF contains binding sites for collagen and platelets to allow platelet plug formation, VWF also binds, transports and interacts with a number of diverse proteins such as FVIII, galectin, vimentin and osteoprotegerin. ${ }^{20-23}$ This 
so-called bus function of VWF is considered responsible for reported pro-apoptotic and pro-inflammatory properties. ${ }^{17}{ }^{24}$ As the role of VWF function in SLE may extend beyond platelet activation, particularly in vascular environments with low shear stress, and VWF-induced platelet aggregation ability in SLE has not been well studied, we investigated the functional state of $\mathrm{VWF}$ and its relation to clinical disease, markers of inflammation and vascular events in SLE.

\section{METHODS}

In a cross-sectional study design, we included 92 patients with SLE from an approved regional lupus disease registry (REK 2015/1400). All fulfilled the relevant American College of Rheumatology's classification criteria for SLE and had detailed clinical information and blood samples collected during an extended outpatient visit. Disease activity was assessed by Systemic Lupus Erythematous Disease Activity Index 2000 (SLEDAI-2K) and organ damage by or Systemic Lupus International Collaborative Clinics (SLICG)-Damage Index (SDI). ${ }^{25} 26$ Thrombotic disease, defined as myocardial infarction, stroke or venous thrombosis, had occurred in 24 patients. Antiphospholipid syndrome was defined as the occurrence of thrombotic or obstetric pathology according to the revised Sapporo criteria during the sustained presence of antiphospholipid (aPL) antibodies. Anticardiolipin antibody (aCL-G and aCL-M; normal levels $<16 \mathrm{IU} / \mathrm{mL}$ ) was analysed by ELISA (Phadia, Freiburg, Germany), while lupus anticoagulant was tested in a phospholipid-dependent coagulation assay. Anti-dsDNA and anti-ENA Ab were measured by immunoassays (Phadia, Freiburg, Germany), while BAFF (B-cell-activating factor), interleukin (IL) 6 and tumour necrosis factor (TNF) $\alpha$ levels (the average of two runs) were determined by quantitative sandwich immunoassay (Single Analyte ELISArrayTM Kit, Super Array, Bioscience, USA). Acute-phase reactants (erythrocyte sedimentation rate (ESR), C-reactive protein (CRP), fibrinogen, ceruloplasmin, albumen and transferrin) were measured through standard assays.

Plasma VWF antigen (VWF:Ag) levels were measured by immunoturbidiometric method (STA-LIA test, Diagnostica, Stago SAS, France), while plasma VWF platelet-binding ability was determined by a ristocetin cofactor assay (VWF:RCo) (Siemens Healthcare Diagnostics Products, Marburg, Germany). Serial dilutions of platelet poor plasma (1:1, 1:2, 1:4, 1:8 and 1:16) were prepared to which a reagent-containing stabilised human-lyophilised platelets and ristocetin were added. After mixing on a glass slide, results were analysed against a dark background with side lighting with the last dilution showing visible agglutination determining the RCo titre of the sample. A reference preparation from pooled plasma was used to produce a standard curve against which the results were read, while diluent was used as a negative control. This assay only measures intrinsic VWF activity as the formalin fixed platelets cannot secrete VWF and was in accordance with the recommendations from the European Pharmacopoeia 5.0. Interassay analytic variation was $<10 \%$ for VWF:RCo and $<3 \%$ for VWF:Ag. To correct for the increase in protein levels, the platelet-aggregating activity per VWF unit was estimated as the VWF RCo/Ag ratio. Plasma factor VIII levels were determined by a functional clotting assay using immunodepleted FVIII plasma (STA-deficient VIII, Diagnostica, Stago SAS, France). All assays were performed in an accredited hospital laboratory and in accordance with the manufactures' recommendations with results expressed as percentage of normal observed in pooled normal plasma. A group of healthy controls ( $\mathrm{n}=16$, mean age 50 years, $77 \%$ female) served as comparators for all serological measurements.

\section{Statistical analysis}

Data are presented as median with IQR or count with percentage, and differences between groups were assessed with non-parametric Mann-Whitney U-test and $\chi^{2}$ test. VWF:Ag levels exceeding the upper range of the assay were assigned the maximum value for computation purposes as further dilutions were not performed (given the lack of diagnostic implications). Multiple linear regression used a backward method with $\mathrm{p}<0.10$ enter and $\mathrm{p}<0.05$ to remain in the model. Resulting $\mathrm{p}$ values $<0.05$ are considered statistically significant. Analysis was performed on IBM SPSS V.22.0.

\section{Ethics}

All participants provided informed and written consent for the use of their confidential data and samples collected as part of a regional registry approved by the regional ethics committee (REK Nord 2015/1400).

\section{RESULTS}

\section{Descriptors}

The clinical characteristics (table 1) and selected laboratory findings for the study cohort (table 2) were typical for a Caucasian SLE cohort with longer standing disease. The levels of VWF:Ag, VWF:RCo and VWF RCo/ $\mathrm{Ag}$ were not associated with age or gender. The levels of VWF:Ag (142\% vs 107\%, p=0.001) and VWF-binding capability (VWF:RCo) $(126 \%$ vs $78 \%, \mathrm{p}=0.041)$ were higher in patients with SLE, but the functional state per unit (VWF RCo/Ag ratio) was no different from controls ( 0.83 vs $0.82, p=0.8$ ) (figure 1 ). There was a strong correlation between VWF:Ag and VWF:RCo (Rs 0.80, $\mathrm{p}<0.001$ ), but not with the $\mathrm{VWF} \mathrm{RCo} / \mathrm{Ag}$ ratio (Rs $-0.13, \mathrm{p}>0.2)$. Factor VIII levels $(149 \%$ vs $115 \%$, $\mathrm{p}=0.046$ ) were increased in patients with SLE, but not fibrinogen levels ( 3.4 vs $3.5 \mathrm{~g} / \mathrm{L}, \mathrm{p}=0.4$ ). Overall, levels for VWF:RCo exceeded the $95 \%$ upper cut-off for healthy controls in 42\%, VWF:Ag levels in 39\% and factor VIII in $42 \%$ of patients. 
Table 1 Clinical descriptors for all patients with SLE at study visit $(n=92)$

\begin{tabular}{|c|c|c|c|c|}
\hline & Overall & $\begin{array}{l}\text { VWF:Ag } \\
\text { (Rs coefficient or } \\
\text { median level) }\end{array}$ & $\begin{array}{l}\text { VWF:RCo } \\
\text { (Rs coefficient or } \\
\text { median level) }\end{array}$ & $\begin{array}{l}\text { VWF RCo/Ag ratio } \\
\text { (Rs coefficient or } \\
\text { median level) }\end{array}$ \\
\hline Age (years) & $49.9(36-60)$ & 0.17 & 0.146 & -0.15 \\
\hline Female gender & $81(88 \%)$ & $141 / 153$ & $120 / 112$ & 0.8 \\
\hline Disease duration (months) & $126(61-213)$ & 0.13 & 0.06 & -0.13 \\
\hline SLEDAI-2K & $6(2-12)$ & 0.18 & -0.06 & 0.18 \\
\hline SLICC-DI & $1(0-2)$ & 0.11 & -0.04 & $-0.25^{\star}$ \\
\hline Prednisone dose (mg/d) & $5(0-7.5)$ & 0.07 & 0.18 & -0.01 \\
\hline BMI & $25(22-28)$ & $0.25^{\star}$ & 0.13 & -0.1 \\
\hline Diastolic blood pressure & $80(68.5-85)$ & 0.12 & 0.06 & -0.15 \\
\hline Renal disease & $45(49 \%)$ & $133 / 143$ & $116 / 126$ & 0.82 \\
\hline Vasculitis & 27 (29\%) & $147 / 142$ & $140 / 120$ & 0.82 \\
\hline Serositis & $21(23 \%)$ & $164 / 142^{*}$ & $140 / 120$ & 0.83 \\
\hline MI, stroke or thrombosis & $24(26 \%)$ & $153 / 141$ & $120 / 120$ & 0.83 \\
\hline aPL antibody presence & 37 (40\%) & $141 / 142$ & $120 / 126$ & 0.81 \\
\hline Hydroxychloroquine user & $56(61 \%)$ & $139 / 149$ & $120 / 140$ & 0.84 \\
\hline Immunosuppressant user & $32(35 \%)$ & $138 / 140$ & $120 / 126$ & 0.82 \\
\hline Aspirin/warfarin user & $31 / 8(34 \% / 9 \%)$ & $140 / 140$ & $120 / 123$ & 0.82 \\
\hline Fish oil, regular user & $39(43 \%)$ & $158 / 142$ & $140 / 120$ & 0.83 \\
\hline \multicolumn{5}{|c|}{$\begin{array}{l}\text { Overall figures represent absolute numbers (\%) or median values (IQR) for the whole cohort. The association of each disease manifestation } \\
\text { with VWF:Ag levels, VWF:RCo and VWF RCo/Ag ratio is given as Rs for continuous variables or as median levels for dichotomous variables } \\
\text { *Statistically significant correlation observed in all patients or a significant difference in level compared with other patients not experiencing } \\
\text { that disease manifestation ( } p<0.05) \text {. } \\
\text { Ag, antigen; aPL, antiphospholipid; BMI, body mass index; MI, myocardial infarction; RCo, ristocetin cofactor; Rs, Spearman rank correlation } \\
\text { coefficients; SLEDAI-2K, Systemic Lupus Erythematous Disease Activity Index 2000; SLICC-DI, Systemic Lupus International Collaborative } \\
\text { Clinics Damage Index; SLE, systemic lupus erythematous; VWF, von Willebrand factor. }\end{array}$} \\
\hline
\end{tabular}

\section{Clinical associations}

Lower VWF:Ag levels were seen in patients with Raynaud's phenomenon ( $130 \%$ vs $148 \%, \mathrm{p}=0.014)$, while increased VWF:Ag levels were seen in patients with serositis $(165 \%$ vs $135 \%, \mathrm{p}=0.013)$. There was an association between VWF:Ag levels and body mass index, but no correlation with SLEDAI-2K, SDI or occurrence of vasculitis, myocardial infarction (MI), stroke, venous thrombosis, lupus nephritis, arthritis, malar rash, smoking or the presence of aPL antibodies (table 1). Two patients $(2.5 \%)$ had abnormally low VWF:RCo $(<50 \%)$, but none had a bleeding tendency. VWF:RCo levels were unrelated to disease manifestations, SLEDAI-2K or SLICC-DI scores. The VWF RCo/Ag ratio was only and inversely related to SDI scores (Rs -0.25 , $\mathrm{p}=0.02)$.

\section{Association with biomarkers}

VWF:Ag levels were significantly associated with ESR (Rs 0.32, $\mathrm{p}=0.002)$, anti-dsDNA Ab (Rs 0.27, $\mathrm{p}<0.01)$, total IgG (Rs $0.33 \mathrm{p}=0.006$ ), fibrinogen (Rs $0.28, \mathrm{p}<0.007$ ), total cholesterol (Rs $0.28, \mathrm{p}=0.007$ ), ceruloplasmin levels (Rs $0.37, \mathrm{p}<0.001$ ) and natural killer cell numbers (Rs $0.21, \mathrm{p}=0.04)$, as well as inversely related to white blood cell (Rs. $-0.26, p<0.05$ ) (table 2). VWF:Ag levels did not correlate with IL-6, TNF- $\alpha$ or BAFF levels (all $p$ values $>0.3)$.

VWF:RCo levels were also significantly associated with ESR (Rs 0.25, p<0.05), anti-dsDNA antibodies (Rs. 0.26, $\mathrm{p}<0.05)$, aspartate amino-transaminase (Rs $0.23, \mathrm{p}<0.05)$ and in addition to total cholesterol (Rs 0.23, p<0.05) and inversely with TIBC $(\mathrm{Rs}-0.29, \mathrm{p}<0.05)$ and transferrin $(\mathrm{Rs}-0.32, \mathrm{p}<0.01)$. No serological correlations were seen for the VWF:RCo/Ag ratio (all $\mathrm{p}>0.2$ ), with the exception of transferrin (Rs $-0.235, \mathrm{p}=0.029$ ).

A multivariate stepwise regression model of statistically significant univariates in SLE patients determined that only FVIII $(p<0.001), \operatorname{IgG}(p=0.003)$ and total cholesterol $(p=0.015)$ were independent predictors for VWF: Ag levels (Table 3). Similarly, only factor VIII $(\mathrm{p}<0.001)$, ceruloplasmin $(\mathrm{p}=0.01)$ and TIBC $(\mathrm{p}=0.001)$ were independent predictors for VWF:RCo levels in patients with SLE, while SLICC-DI $(\mathrm{p}=0.005)$ and transferrin levels $(\mathrm{p}=0.01)$ were independent predictors for VWF RCo/Ag levels.

\section{DISCUSSION}

This study demonstrates that in spite of the presence of higher VWF protein levels and functional ability (VWF: RCo) in patients with SLE, there was no increased propensity for platelet aggregation per unit of VWF and no association with thrombotic events. Upregulated VWF:Ag and VWF:RCo correlated with a range of acute-phase reactants including IgG and anti-dsDNA antibody, ceruloplasmin and TIBC levels. Together, these results indicate that increased VWF levels in SLE mirror immune-mediated inflammation rather than a state of increased platelet activation.

Sporadic cases of acquired VWD have been reported in patients with SLE, where bleeding resulted from 
Table 2 Laboratory findings in all patients with SLE $(n=92)$

\begin{tabular}{|c|c|c|c|c|}
\hline & Median levels & $\begin{array}{l}\text { VWF:Ag } \\
\text { Rs. }\end{array}$ & $\begin{array}{l}\text { VWF:RCo } \\
\text { Rs. }\end{array}$ & $\begin{array}{l}\text { VWF RCo/Ag ratio } \\
\text { Rs. }\end{array}$ \\
\hline Factor VIII & $149 \%(121-160)^{*}$ & $0.56 \dagger$ & $0.53 \dagger$ & -0.02 \\
\hline Fibrinogen & $3.5(3.2-4.1)$ & $0.28 \ddagger$ & 0.203 & -0.08 \\
\hline $\mathrm{ESR}(\mathrm{mm} / \mathrm{h})$ & $20(10-33)^{\star}$ & $0.32 \dagger$ & $0.26 \ddagger$ & 0.04 \\
\hline CRP (mg/L) & $4.0(4.0-4.0)$ & 0.13 & 0.136 & 0.03 \\
\hline $\mathrm{Hb}(\mathrm{g} / \mathrm{dL})$ & $13.0(12.1-14.1)$ & 0.10 & -0.106 & -0.12 \\
\hline WBC $\left(\times 10^{9} / \mathrm{L}\right)$ & $5.8(4.3-7.1)^{\star}$ & $-0.26 \ddagger$ & -0.15 & -0.17 \\
\hline Lymphocytes (×109/L) & $1.00(1.00-2.00)^{*}$ & 0.11 & 0.05 & -0.02 \\
\hline NK cells & $0.10(0.7-0.18)$ & $0.21 \ddagger$ & 0.17 & -0.11 \\
\hline B cells & $0.11(0.04-0.25)$ & 0.06 & 0.04 & -0.04 \\
\hline Platelets & 256 (217-295) & -0.1 & -0.11 & -0.02 \\
\hline Albumen & $43(41-45)^{\star}$ & 0.15 & -0.19 & -0.06 \\
\hline Creatinine (mmol/L) & $63(53-70)$ & 0.11 & 0.14 & -0.06 \\
\hline Anti-dsDNA Ab (IU) & $120(90-170)^{*}$ & $0.27 \dagger$ & $0.26 \ddagger$ & 0.05 \\
\hline C3 & $0.95(0.79-1.11)$ & 0.18 & 0.04 & -0.15 \\
\hline Cholesterol & $4.6(4.0-5.3)$ & $0.28 \dagger$ & $0.23 \ddagger$ & -0.03 \\
\hline $\lg G$ & 13.3 (10.9-16.3) & $0.33 \dagger$ & 0.171 & 0.04 \\
\hline IL-6 (pg/mL) & $14(14-19)$ & 0.06 & 0.12 & 0.27 \\
\hline $\mathrm{TNF} \alpha(\mathrm{pg} / \mathrm{mL})$ & $34.3(21-87)$ & 0.04 & 0.07 & 0.08 \\
\hline BAFF (pg/mL) & $1740(1280-2351)^{*}$ & 0.08 & 0.19 & 0.15 \\
\hline Prealbumin & $0.25(0.22-0.29)$ & -0.07 & -0.06 & 0.08 \\
\hline Ceruloplasmin & $0.32(0.29-0.38)$ & $0.40 \dagger$ & $0.37 \dagger$ & 0.02 \\
\hline TIBC & 59.5 (53.0-67.0) & -0.12 & $-0.29 \ddagger$ & $-0.25 \ddagger$ \\
\hline Transferrin & $2.40(2.10-2.65)$ & -0.14 & $-0.32 \dagger$ & $-0.23 \ddagger$ \\
\hline
\end{tabular}

Figures are given as median values (IQR) observed in the whole cohort and as the Spearman rank correlation coefficients (Rs) for the association between each serological marker and VWF:Ag levels, VWF:RCo and VWF RCo/Ag ratio.

*Statistically significant different level compared with healthy controls $(p<0.05)$.

†Statistically significant correlation $(p<0.01)$.

¥Statistically significant correlation $(p<0.05)$.

Ag, antigen; BAFF, B-cell-activating factor; CRP, C-reactive protein; ESR, erythrocyte sedimentation rate; Hb, haemoglobin; IL, interleukin; NK cells, natural killer cells; RCo, ristocetin cofactor; Rs, Spearman rank correlation coefficients; SLE, systemic lupus erythematous; TIBC, total iron-binding capacity; TNF, tumour necrosis factor; VWF, von Willebrand Factor, WBC, white blood cell.

antibodies against VWF causing a reduction in the level or function of VWF. In most cases, immunomodulating treatment restored VWF levels and activity and resolved bleeding. ${ }^{27-31}$ Even though two patients in our cohort had abnormally low VWF:RCo, the decreases were moderate only (VWF:RCo $36 \%$ and $42 \%$, respectively) and were not associated with a bleeding diathesis. Thus, while we cannot exclude the presence of functional antibodies against VWF in these patients, at the time of study-acquired VWD was not present in this cohort.

Our results are in line with a number of other studies showing higher VWF levels in patients with SLE than in controls, but a direct comparison of VWF levels between studies is confounded by the use of different VWF assays. ${ }^{4}{ }^{15-17}$ The reason for and the functional consequences of increased VWF level in patients with SLE are not well understood. VWF is exclusively synthesised in endothelial cells and megakaryocytes, and while it is not currently possible to reliably determine the site of VWF production, we found no associations between platelet counts, ongoing or historical vasculitis and VWF levels. However, we did not examine endothelial function or bone marrow in our patients. Similar to other studies in Caucasian cohorts, we could not establish a relation between overall clinical disease activity and VWF:Ag levels, ${ }^{17} 32$ although such a relation has been reported in African-American patients. ${ }^{15}$

To the best of our knowledge, data on VWF platelet aggregation activity in SLE have not been published, but the fact that VWF platelet aggregation activity in patients with SLE is similar to that in controls despite higher VWF:Ag levels strongly suggests that the predictive value of VWF levels for a first arterial event in SLE must be attributed to other characteristics of VWF. ${ }^{511}{ }^{18}$ Platelet aggregation by VWF is strongly dependent on its molecular weight, which is mainly determined by its multimeric composition. ${ }^{1}$ Large and intermediate multimers of plasma VWF are more active in platelet aggregation than dimers and may be more frequent in patients with SLE with vascular complications. ${ }^{33} 34$ This indicates that VWF multimer analysis, possibly in combination with ADAMTS13 levels that determine VWF size, could be a more relevant assay for assessing VWF-related thrombotic risk in SLE than VWF protein levels.

Furthermore, VWF has other roles in addition to platelet and FVIII binding. VWF closely follows CRP levels after bacterial infection suggesting it is involved in the acute-phase response. ${ }^{35}$ The significant correlations 

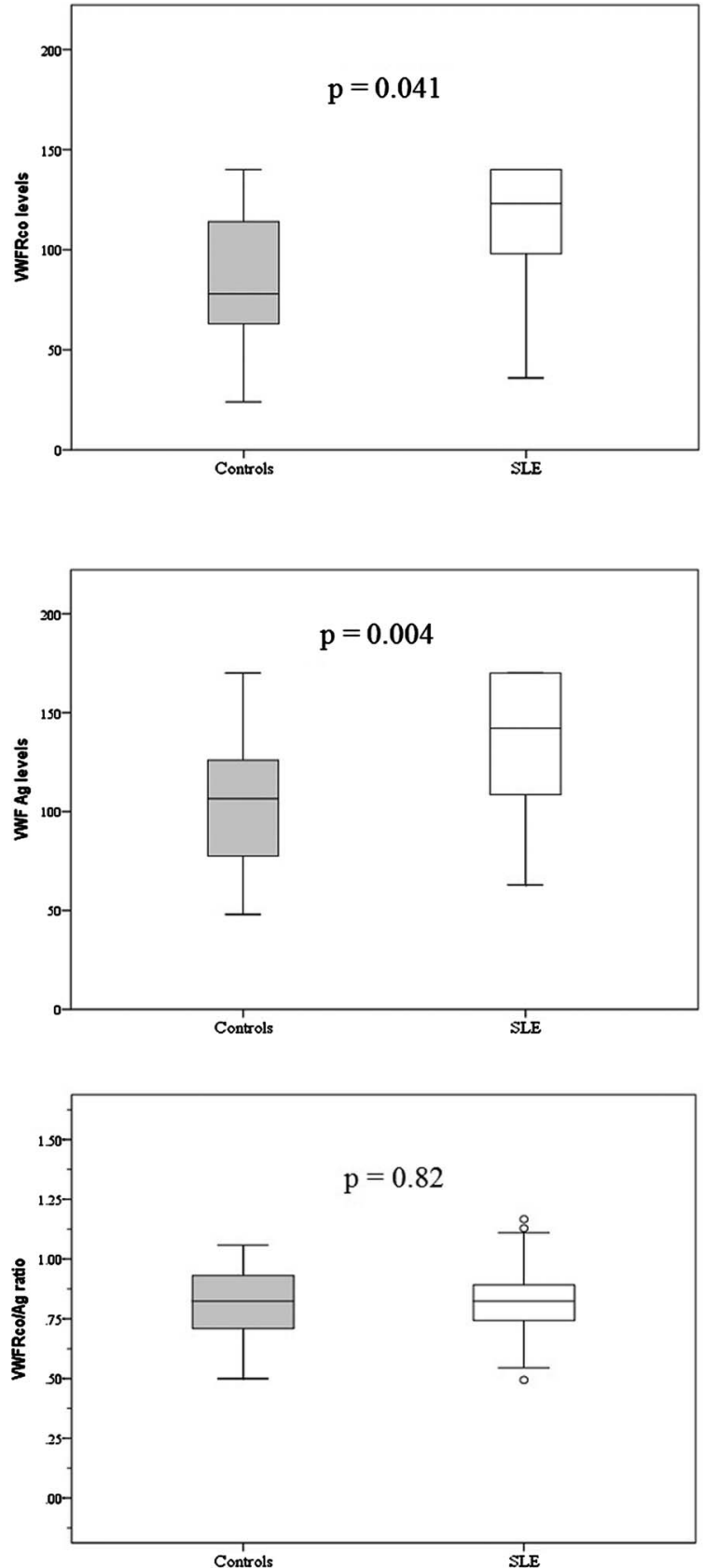

Figure 1 Platelet-binding capability (VWF:RCo), VWF levels (VWF:Ag) and functional state per VWF unit (VWF RCo/Ag ratio) in controls and patients with SLE. Ag, antigen; RCo, ristocetin cofactor; SLE, systemic lupus erythematous; VWF, von Willebrand factor. between VWF levels and a range of acute-phase proteins (ESR, fibrinogen, ceruloplasmin, prealbumin) shown herein, confirms that VWF acts as an acute-phase reactant in patients with SLE as well. Although these do not prove causality, they indicate that VWF levels follow immune-mediated inflammatory response in this SLE cohort. The lack of correlation between VWF and CRP levels we found is less surprising given the aberrant behaviour of CRP in SLE. ${ }^{36}$ The strong and independent association between VWF and overall IgG levels in addition to a correlation with disease-specific anti-dsDNA antibody levels observed in this study indicates that the VWF secretion in SLE is closely linked to the autoimmune setting of the acute-phase response. This supports findings by Lai $e t a l,{ }^{37}$ who showed that IgG fractions containing antibodies to DNA increases the in vitro release of VWF from human vascular endothelium. Curiel et $\mathrm{al}^{15}$ also demonstrated an inverse association between VWF and C3 levels in patients with SLE, providing further support for the idea that complement consumption, which is almost invariable due to immune complex activation in SLE, leads to VWF release. More recently a size dependent role for $\mathrm{VWF}$ was described for complement regulation, as normal-sized VWF multimers inhibited complement activation, while large VWF multimers permitted complement activation. ${ }^{38}$ A recent paper reported normal multimer analysis in patients with SLE in the face of lower ADAMTS13 levels and higher VWF:Ag than controls, which supports their behaviour as acute-phase reactants rather than direct prothrombotic factors. ${ }^{39}$

Acute-phase reactant production by hepatocytes is mainly mediated through the effects of IL-6, but we were unable to demonstrate a significant relation between VWF levels and levels of the pro-inflammatory cytokines IL-6, TNFa and BAFF (table 2). A post hoc analysis suggested that VWF levels tended to be higher in anti-dsDNA-positive patients with increased $(>20 \mathrm{pg} /$ $\mathrm{mL}$ ) IL-6 levels ( $158 \%$ vs $137 \%, \mathrm{p}=0.06)$. However, from our patient cohort, we cannot confirm the findings of in vitro studies in which pro-inflammatory cytokinemediated VWF release. ${ }^{40} 41$

We observed a surprising inverse correlation between $\mathrm{SDI}$ and $\mathrm{RCo} / \mathrm{Ag}$, which is the opposite of what would have been expected. While the VWF RCo/Ag ratio was not significantly different between patients on or off

Table 3 Multivariate stepwise regression for significant univariates in SLE patients

\begin{tabular}{llcrrr}
\hline DV & IV & B & Lower 95\% & Upper 95\% & p-value \\
\hline VWF Antigen & Factor VIII & 0.57 & 0.36 & 0.78 & $<0.001$ \\
& Cholesterol & 6.55 & 1.32 & 0.015 \\
& IgG & 2.11 & 0.74 & 3.47 & 0.003 \\
VWF Ristocetin Cofactor & Factor VIII & 0.57 & 0.39 & 0.74 & 152.04 \\
& Ceruloplasmin & 86.76 & 21.48 & -0.39 & 0.010 \\
VWF RCo/Ag & TIBC & -0.88 & -1.37 & -0.02 & 0.001 \\
& Transferrin & -0.09 & -0.16 & -0.01 & 0.010 \\
\hline
\end{tabular}


anticoagulation treatment (data not shown), we cannot exclude such an influence in patients with prior vascular disease as they had higher median SDI scores ( 1.9 vs 0.9 , $\mathrm{p}=0.02$ )

Hydroxychloroquine treatment is common place in SLE, as it reduces relapse rates and the risk of thrombotic events, ${ }^{31}$ and in experimental lupus models, the drug has been found to reduce endothelial dysfunction. ${ }^{14}{ }^{42}$ We did not find a significant effect of sustained hydroxychloroquine therapy on of VWF levels or platelet aggregation activity in our cohort, suggesting that hydroxychloroquine exerts its vasoprotective effects through other pathways. Similarly, we were unable to detect an effect of antiphospholipid antibody presence or the use of anticoagulant drugs on VWF:Ag or functional activity.

The restrictions of this study must be recognised. These include the cross-sectional design, which limits the validity of our finding to a single observation point during a chronic disease. The inclusion of a measure of endothelial stunning could have strengthened our findings further and low numbers in subgroup analyses may have masked significant findings. Furthermore, while the assays used are validated for diagnosing VWD due to reduced VWF levels and/or activity, there is no evidence that they are valid markers in evaluating thrombotic risk. Finally, MI, stroke and venous thrombosis most likely have different underlying pathology, but too few such patients were included in the current study for these events to be analysed separately. The strength of our study lies in the comprehensive and simultaneous analysis of clinical and serological data from a well-defined homogenous cohort and the first time inclusion of a functional VWF assay.

In conclusion, increased VWF:Ag levels mirrored acute-phase reactants including anti-dsDNA levels in this SLE cohort, but as there was no relative increase in the functional activity per unit (VWF $\mathrm{RCo} / \mathrm{Ag}$ ), this indicates that there was no propensity for increased VWF-induced platelet aggregation in SLE.

Acknowledgements The technical support by Kirsten Nilsen and Andrea Becker Merok is greatly acknowledged.

Contributors JCN designed the study, supervised data collection and participated in data analyses and final manuscript drafting. WDR performed data analyses and was actively involved in final manuscript drafting. GØE helped design the study, performed data collection and critically reviewed the final manuscript.

Funding JCN is supported by an unrestricted grant of Arthritis Foundation of Werster Australia.

Competing interests None declared.

Patient consent Obtained.

Ethics approval The disease registry with de-identified collection data was approved by the Regional Ethics Committee of Northern Norway (REK 2015/1400).

Provenance and peer review Not commissioned; externally peer reviewed.

Data sharing statement The ethics approval does not allow for sharing of these data without prior review by the Regional Ethics Committee of Northern Norway. Please contact the authors for information on procedures.
Open Access This is an Open Access article distributed in accordance with the Creative Commons Attribution Non Commercial (CC BY-NC 4.0) license, which permits others to distribute, remix, adapt, build upon this work noncommercially, and license their derivative works on different terms, provided the original work is properly cited and the use is non-commercial. See: http:// creativecommons.org/licenses/by-nc/4.0/

\section{REFERENCES}

1. Chan $\mathrm{CH}$, Pieper IL, Fleming $\mathrm{S}$, et al. The effect of shear stress on the size, structure, and function of human von Willebrand factor. Artif Organs 2014;38:741-50.

2. Mourik MJ, Valentijn JA, Voorberg J, et al. von Willebrand factor remodeling during exocytosis from vascular endothelial cells. J Thromb Haemost 2013;11:2009-19.

3. Spiel AO, Gilbert JC, Jilma B. von Willebrand factor in cardiovascular disease: focus on acute coronary syndromes. Circulation 2008;117:1449-59.

4. Favaloro EJ. Diagnosis and classification of von Willebrand disease: a review of the differential utility of various functional von Willebrand factor assays. Blood Coagul Fibrinolysis 2011;22:553-64.

5. Paulinska $P$, Spiel A, Jilma B. Role of von Willebrand factor in vascular disease. Hamostaseologie 2009;29:32-8.

6. Mannucci PM, Vanoli M, Forza I, et al. Von Willebrand factor cleaving protease (ADAMTS-13) in 123 patients with connective tissue diseases (systemic lupus erythematosus and systemic sclerosis). Haematologica 2003;88:914-18.

7. Lee YJ, Shin KC, Kang SW, et al. Type III procollagen N-terminal propeptide, soluble interleukin-2 receptor, and von Willebrand factor in systemic sclerosis. Clin Exp Rheumatol 2001;19:69-74.

8. Sonneveld MA, de Maat MP, Leebeek FW. Von Willebrand factor and ADAMTS13 in arterial thrombosis: a systematic review and meta-analysis. Blood Rev 2014;28:167-78.

9. Vikerfors A, Johansson AB, Gustafsson JT, et al. Clinical manifestations and anti-phospholipid antibodies in 712 patients with systemic lupus erythematosus: evaluation of two diagnostic assays. Rheumatology (Oxford) 2013;52:501-9.

10. Steyers CM III, Miller FJ, Jr. Endothelial dysfunction in chronic inflammatory diseases. Int J Mol Sci 2014;15:11324-49.

11. Gustafsson JT, Svenungsson E. Definitions of and contributions to cardiovascular disease in systemic lupus erythematosus. Autoimmunity 2014;47:67-76.

12. Horstman LL, Jy W, Jimenez JJ, et al. Endothelial microparticles as markers of endothelial dysfunction. Front Biosci 2004;9:1118-35.

13. Yildiz A, Soydinc S. Diastolic dysfunction and endothelial dysfunction in systemic lupus erythematosus. Rheumatol Int 2015;35:1281-2.

14. Virdis A, Tani C, Duranti E, et al. Early treatment with hydroxychloroquine prevents the development of endothelial dysfunction in a murine model of systemic lupus erythematosus. Arthritis Res Ther 2015;17:277.

15. Curiel RV, Bhagati R, Basavaraju L, et al. Von Willebrand factor, red cell fragmentation, and disease activity in systemic lupus erythematosus. HSS J 2008;4:170-4.

16. Matsuda J, Kawasugi K, Gohchi K, et al. Clinical significance of the venous occlusion test on systemic lupus erythematosus patients with a focus on changes in blood levels of tissue plasminogen activator, von Willebrand factor antigen, and thrombomodulin. Acta Haematol 1992:88:22-6.

17. de Leeuw K, Freire B, Smit AJ, et al. Traditional and non-traditional risk factors contribute to the development of accelerated atherosclerosis in patients with systemic lupus erythematosus. Lupus 2006;15:675-82.

18. Gustafsson J, Gunnarsson I, Börjesson O, et al. Predictors of the first cardiovascular event in patients with systemic lupus erythematosus-a prospective cohort study. Arthritis Res Ther 2009;11:R186.

19. van Schie MC, van Loon JE, de Maat MP, et al. Genetic determinants of von Willebrand factor levels and activity in relation to the risk of cardiovascular disease: a review. J Thromb Haemost 2011;9:899-908.

20. Da Q, Behymer M, Correa Jl, et al. Platelet adhesion involves a novel interaction between vimentin and von Willebrand factor under high shear stress. Blood 2014;123:2715-21.

21. Shahbazi S, Lenting PJ, Fribourg C, et al. Characterization of the interaction between von Willebrand factor and osteoprotegerin. J Thromb Haemost 2007;5:1956-62.

22. Zannettino AC, Holding CA, Diamond $\mathrm{P}$, et al. Osteoprotegerin (OPG) is localized to the Weibel-Palade bodies of human vascular 
endothelial cells and is physically associated with von Willebrand factor. J Cell Physiol 2005;204:714-23.

23. Saint-Lu N, Oortwijn BD, Pegon JN, et al. Identification of galectin-1 and galectin-3 as novel partners for von Willebrand factor. Arterioscler Thromb Vasc Biol 2012;32:894-901.

24. Baud'huin M, Duplomb L, Téletchéa S, et al. Factor VIII-von Willebrand factor complex inhibits osteoclastogenesis and controls cell survival. J Biol Chem 2009;284:31704-13.

25. Touma Z, Urowitz MB, Gladman DD. SLEDAI-2K for a 30-day window. Lupus 2010;19:49-51.

26. Gladman DD, Goldsmith $\mathrm{CH}$, Urowitz MB, et al. The Systemic Lupus International Collaborating Clinics/American College of Rheumatology (SLICC/ACR) Damage Index for Systemic Lupus Erythematosus International Comparison. J Rheumatol 2000;27:373-6.

27. Hong $\mathrm{S}$, Lee J, Chi H, et al. Systemic lupus erythematosus complicated by acquired von Willebrand's syndrome. Lupus 2008;17:846-8.

28. Güngör T, Furlan M, Lämmle B, et al. Acquired deficiency of von Willebrand factor-cleaving protease in a patient suffering from acute systemic lupus erythematosus. Rheumatology (Oxford) 2001:40:940-2.

29. Igarashi N, Miura M, Kato E, et al. Acquired von Willebrand's syndrome with lupus-like serology. Am J Pediatr Hematol Oncol 1989;11:32-5.

30. Soff GA, Green D. Autoantibody to von Willebrand factor in systemic lupus erythematosus. J Lab Clin Med 1993;121:424-30.

31. Xiong W, Lahita RG. Pragmatic approaches to therapy for systemic lupus erythematosus. Nat Rev Rheumatol 2014;10:97-107.

32. Constans J, Dupuy R, Blann AD, et al. Anti-endothelial cell autoantibodies and soluble markers of endothelial cell dysfunction in systemic lupus erythematosus. J Rheumatol 2003;30:1963-6.

33. Kagawa H, Okubo S, Yasunaga K. Plasma coagulation and fibrinolysis parameters in patients with collagen diseases, and analysis of the multimeric structure of von Willebrand factor (VWF). Int J Hematol 1991;54:429-39.

34. Kagawa $\mathrm{H}$, Okubo $\mathrm{S}$, Iwata $\mathrm{K}$, et al. Addition of serum from patients with collagen diseases increases to cultured human endothelial cells production and release of von Willebrand factor. Am J Hematol 1993;42:309-15.

35. Pottinger BE, Read RC, Paleolog EM, et al. von Willebrand factor is an acute phase reactant in man. Thromb Res 1989;53: 387-94.

36. Enocsson $\mathrm{H}$, Sjöwall $\mathrm{C}$, Kastbom A, et al. Association of serum C-reactive protein levels with lupus disease activity in the absence of measurable interferon-alpha and a C-reactive protein gene variant. Thromb Res 2014;66:1568-73.

37. Lai KN, Leung JC, Lai KB, et al. Increased release of von Willebrand factor antigen from endothelial cells by anti-DNA autoantibodies. Ann Rheum Dis 1996;55:57-62.

38. Feng $\mathrm{S}$, Liang $\mathrm{X}$, Kroll $\mathrm{MH}$, et al. von Willebrand factor is a cofactor in complement regulation. Blood 2015;125:1034-7.

39. Martin-Rodriguez S, Reverter JC, Tàssies D, et al. Reduced ADAMTS13 activity is associated with thrombotic risk in systemic lupus erythematosus. Lupus 2015;24:1143-9.

40. Bernardo A, Ball C, Nolasco L, et al. Effects of inflammatory cytokines on the release and cleavage of the endothelial cell-derived ultralarge von Willebrand factor multimers under flow. Blood 2004;104: $100-6$.

41. Thacker SG, Duquaine D, Park J, et al. Lupus-prone New Zealand Black/New Zealand White F1 mice display endothelial dysfunction and abnormal phenotype and function of endothelial progenitor cells. Lupus 2010;19:288-99.

42. Gómez-Guzmán M, Jiménez R, Romero M, et al. Chronic hydroxychloroquine improves endothelial dysfunction and protects kidney in a mouse model of systemic lupus erythematosus. Hypertension 2014;64:330-7. 